\title{
Analysis of airplane signatures using passive VHF radar for recognition perspectives
}

\author{
Hinostroza Sáenz, Israel D. \\ SONDRA, CentraleSupélec \\ Gif-sur-Yvette, France \\ israel.hinostroza@centralesupelec.fr
}

\author{
Ren, Chengfang \\ SONDRA, CentraleSupélec \\ Gif-sur-Yvette, France \\ chengfang.ren@centralesupelec.fr \\ Fix, Jérémy \\ CentraleSupélec \\ Metz, France \\ jeremy.fix@ centralesupelec.fr
}

\author{
Letertre, Thierry \\ SONDRA, CentraleSupélec \\ Gif-sur-Yvette, France \\ thierry.letertre@centralesupelec.fr
}

\begin{abstract}
Radio beacons, at around $115 \mathrm{MHz}$ (VHF, VOR), are used as opportunity donors in a passive radar configuration. Full-wave simulation of three categories (according to size) of aircrafts shows that their bistatic RCS are statistically comparable without any additional information but perform differently in time (while flying). This discrepancy can be exploited to recognize the aircrafts among the categories. Measurements confirm these ideas and indicate that it may be possible to differentiate the aircrafts among the same category. Encouraging initial results were obtained using Convolutional Neural Networks to classify airplane categories combining simulated bistatic RCS results and real trajectories (collected from ADS-B data).
\end{abstract}

Keywords-RCS, bistatic, passive radar, VHF, full-wave simulation, aircraft

\section{INTRODUCTION}

To facilitate the flight navigation, radio beacons, as VORs (VHF Omnidirectional Range) since 1940's, have been installed around the world. The main advantage of VORs is that they transmit an omnidirectional signal, horizontal polarization, in the VHF band $(108-118 \mathrm{MHz})$ [1] [2] making it ideal, as opportunity sources, for passive radar applications [3] [4]. In this band the wavelength is about $2.6 \mathrm{~m}$ which means that the size of commercial airplanes is on the order of tens of wavelengths. By exploiting the Automatic Dependent Surveillance-Broadcast (ADS-B) data broadcasted (L band) by the airplanes, the position of the airplane can be known and its radar cross section (RCS) can be obtained, as in [3], and it is of the order of tens of dBsm. Work has also been done to classify, according to size (small, medium, large), the airplanes by exploiting an aggregation of RCS of the airplanes [4] [5]. Contrary to optical recognition, characterization of an airplane by its RCS signature is challenging in VHF band, but independent of daytime and weather.

In this paper, we highlight, through simulation and measurements, discriminant RCS patterns for different sizes of planes. First, we present some simulation results of RCS of three airplanes (small, medium, large) in order to understand better their similarities and differences. The second part presents the measurement setup and collected signals, showing a good agreement with the simulation results. The third part focus on the classification of airplanes using convolutional neural networks $(\mathrm{CNN})$ with $2 \mathrm{D}$ images (RCS values vs. aspect and bistatic angle). This representation seems to be relevant based on the obtained results. Finally, some conclusions and perspectives are presented.
TABLE I. AIRPLANE MODELS DIMENSIONS

\begin{tabular}{|l|c|c|c|}
\hline Model size & Wingspan $[\mathbf{m}]$ & Length $[\mathbf{m}]$ & \# total of motors \\
\hline Small & 24 & 29 & 2 \\
\hline Medium & 33.5 & 40 & 2 \\
\hline Large & 51 & 60 & 4 \\
\hline
\end{tabular}

\section{MODELling AND SimUlATION OF AIRPLANES}

\section{A. Airplane models}

The dimensions and number of motors of each airplane model can be seen in the Tab. I (see Fig. 1 for a scale). The dimensions of the large, medium and small models, respectively. Perfect electrical conductor (PEC) was considered for the models.

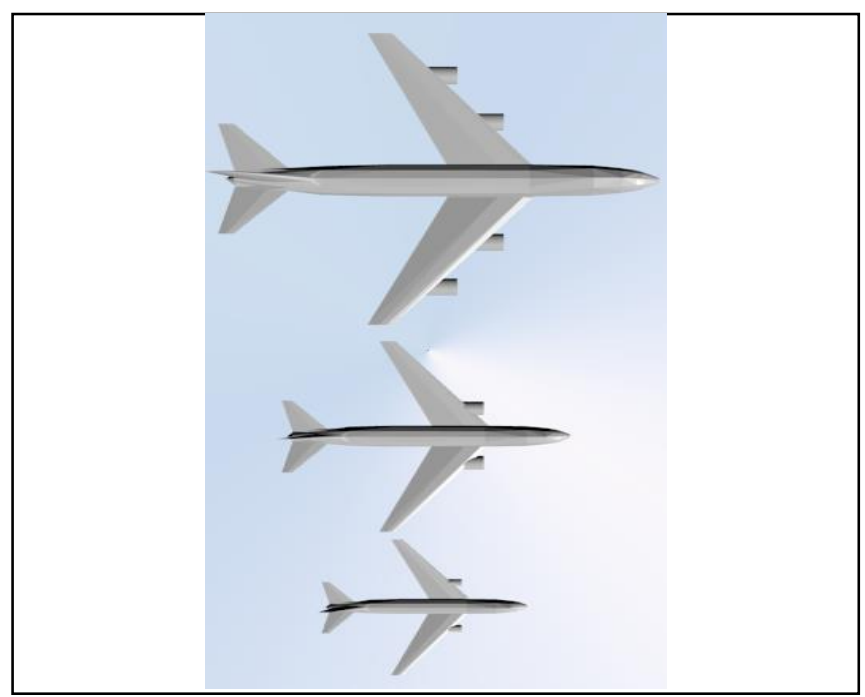

Fig. 1. From top to bottom: large, medium and small airplane models used in FEKO.

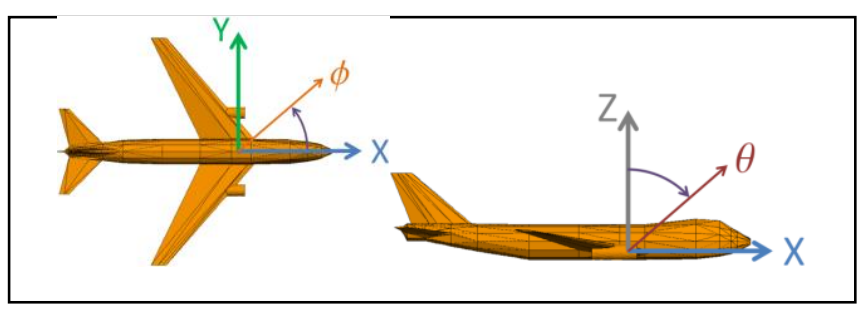

Fig. 2. Simulation setup of airplane models, PEC, in FEKO and definition of spherical coordinates used in the simulations. 


\section{B. Simulation results - RCS}

A full-wave simulation using method of moments (MoM) was carried out in FEKO. See Fig. 2 for the simulation setup and spherical coordinates used for the simulation. An incident horizontally polarized (along $\varphi$ axis) plane wave was considered with incident direction $\left(\theta_{\mathrm{i}}, \varphi_{\mathrm{i}}\right) \in[90,180] \times$ $[0,180]$ (due to the symmetry of the planes, incident angles $\varphi_{\mathrm{i}}$ larger than 180 are obtained from the original simulations) and scattered angles $\left(\theta_{\mathrm{s}}, \varphi_{\mathrm{s}}\right) \in[0,180] \times[0,360]$, in both cases with angular step of 1deg. Fig. 3 shows the histograms of bistatic RCS of the airplanes models (small, medium and large) for $\mathrm{HH}(\varphi-\varphi)$ and $\mathrm{HV}(\varphi-\theta)$ cases and for all scattered and incident angles considered lines above. We can see that most of the RCS values are concentrated between $0 \mathrm{dBsm}$ and $40 \mathrm{dBsm}$ (in accordance with [3], Airbus 321 is comparable to the model of medium size used lines above). Most importantly, in global, RCS values between the airplanes are very similar, making the discrimination difficult from RCS only. Additionally, there is no much difference, in global, between RCS values for the $\mathrm{HH}$ and HV cases. Since the VOR transmitter uses $\mathrm{H}$ polarization, a reception using V polarization helps to strongly reduce the direct path.

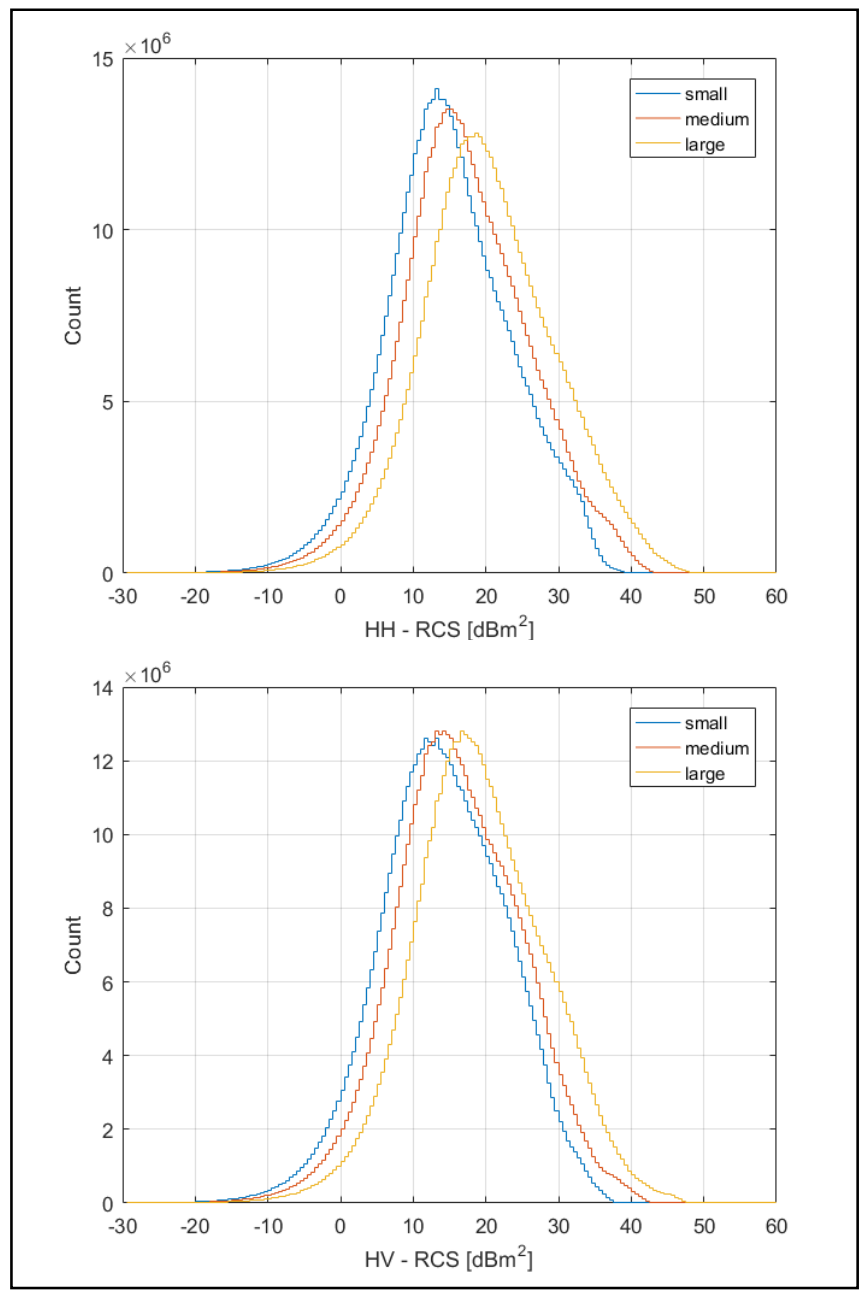

Fig. 3. Histograms of bistatic RCS (dBsm) obtained by simulation, HH ( $\varphi$ $\varphi$, top) and $\mathrm{HV}(\varphi-\theta$, bottom), for small, medium and large airplane models.

\section{Simulation results - Power time series}

Using the simulation results and ADS-B data from the airplanes, we can also synthesize the variation of the bistatic RCS of the planes for the trajectories of the planes. For this case, we consider a passive radar configuration where the transmitter (Tx) is the RBT VOR (close to Rambouillet), and the receiver $(\mathrm{Rx})$ is near CentraleSupélec - Gif.

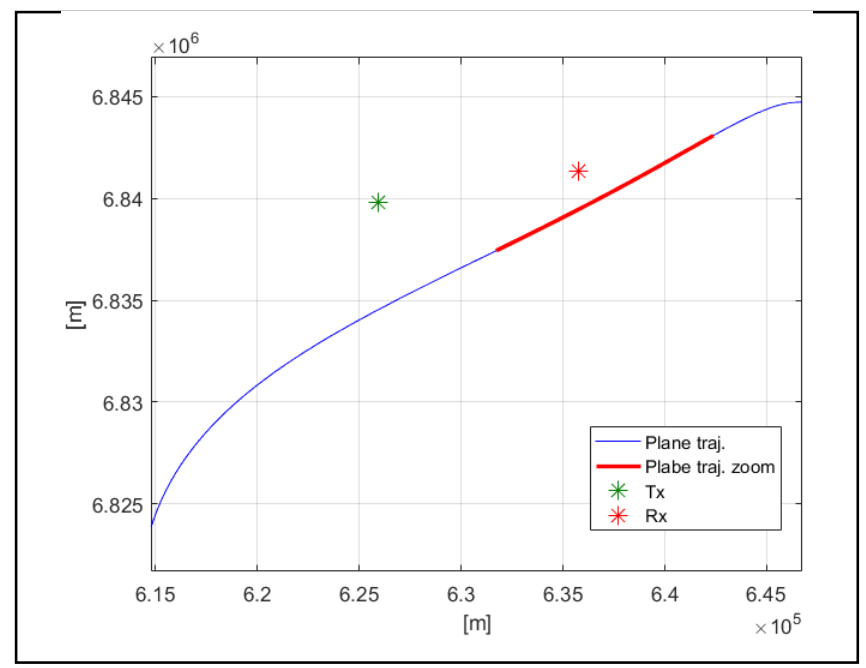

Fig. 4. Position of Tx (RBT VOR) in green, Rx (near CentraleSupélecGif) in red, plane's trajectory in blue (ADS-B data for 6.3 minutes) and "zoom" of trajectory in thick red line (between timestamps 3 and $5 \mathrm{~min}$ ). The plane is moving from left to right. Lambert 93 coordinates (meters)
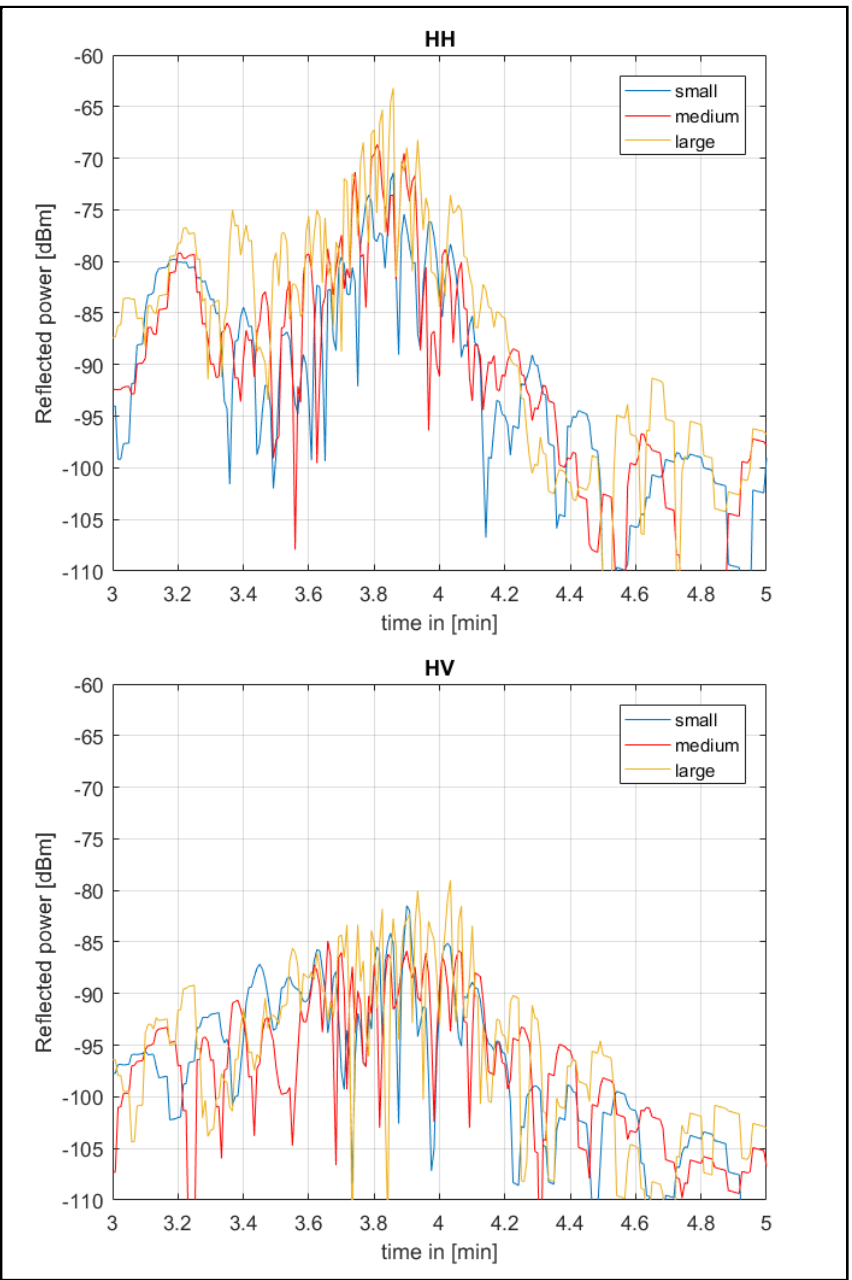

Fig. 5. Synthesis of received power ( $\mathrm{HH}$ and $\mathrm{HV}$, from top to bottom, respectively) near CentraleSupélec, for $2 \mathrm{~min}$, considering an emitted power of $40 \mathrm{dBW}$ by RBT VOR. The position of the plane, considered for this synthesis, is the one labeled as "zoom" (thick red line) in Fig. 4. 
Fig. 4 shows the position of the $\mathrm{Tx}, \mathrm{Rx}$, the plane's trajectory (obtained from real ADS-B data for about 6 minutes) and a "zoom" version of the trajectory (between 3.5 and 5.5 minutes), all of them in Lambert 93 coordinates (spatial units in meters). The plane moves from left to right (approaching landing at Orly airport). Using this "single" trajectory, the received power (considering a transmitting power of $40 \mathrm{dBW}$ ) versus time (in min, $2 \mathrm{~Hz}$ of sampling) is shown for the three planes and polarizations ( $\mathrm{HH}, \mathrm{HV})$ in Fig. 5.

From Fig. 5, we can see that the ranges of values of received power, per polarization, are similar (as expected from the histograms, cf. Fig. 3) besides a peak of $5 \mathrm{~dB}$ (around $3.85 \mathrm{~min}$ ) above the rest of the other cases, which corresponds to the large airplane. The RCS (not shown) for all the cases is around $30 \mathrm{dBsm}$ for $\mathrm{HH}$ polarization and 15 $\mathrm{dBsm}$ for $\mathrm{HV}$ polarization.

What is different, globally, is the frequency of fluctuation of the received power (see Fig. 5), which is different according to the size of the airplane: high frequency for large plane, medium frequency for medium plane and low frequency for small plane, and for both polarizations. This is somewhat expected since, due to the movement of plane, the space of bistatic RCS of the planes is swept with the same rate. The frequency of fluctuations indicates, in this case, the angular frequency due to sweeping throughout the peaks (width and number) of the scattered power due to the illumination of the object: large objects will induce more and finer peaks compared to smaller objects.

We can also see that the received power for the $\mathrm{HH}$ polarization is about $15 \mathrm{~dB}$ higher than the $\mathrm{HV}$ polarization (this might not always be the case, see histograms, Fig. 3). We note that in all the cases we have used a single trajectory and single movement dynamics (same speed, orientation), hence the frequency of fluctuations of RCS (or received power) in real life will depend on the speed of the planes. In order to avoid this problem an estimation of the position of the airplane may be used to transform the frequency of fluctuations versus time into angular frequency.

\section{MEASUREMENTS}

\section{A. Measurement setup}

To collect the scattered power from the airplane, a receiver was installed close to CentraleSupélec-Gif. A VHF antenna with horizontal polarization was used. A SDR receiver was also used to collect complex signals, centered at VOR frequency $(114.7 \mathrm{MHz})$, and connected to a PC (commanded by a Python script). An ADS-B decoder was also used to retrieve the trajectory of the airplane and type of the airplane. Note that the trajectories of the planes (for this data collection) are very similar: approaching Orly-airport.

\section{B. Examples of Collected data}

Fig. 6 shows the evolution of relative received power (after pre-processing) in $\mathrm{dB}$ for 4 airplanes and two categories: small and medium. The planes are approaching Orly-airport, and, hence, following a similar flight path (although not exactly same path, speed, and attitude). The received power in Fig. 6 contains the reflected signal on the airplane (from RBT VOR) and the "direct" path but strongly attenuated (level of about $-66 \mathrm{~dB}$ ) due to losses in the propagation.

From Fig. 6 we can appreciate the differences on the power evolution between categories: in amplitude in general, as well as in relative level between the main peak (highest level) and secondary ones (close to the main peak). At this stage, we recall that we cannot compare the frequency of fluctuations on the received power due to the different speeds of the plane. It can also be seen that there are clearly differences between the two airplanes of the category small. Note as well that the power evolutions related to the medium category (Boeing 737) are very similar since they have similar flight path and attitude.

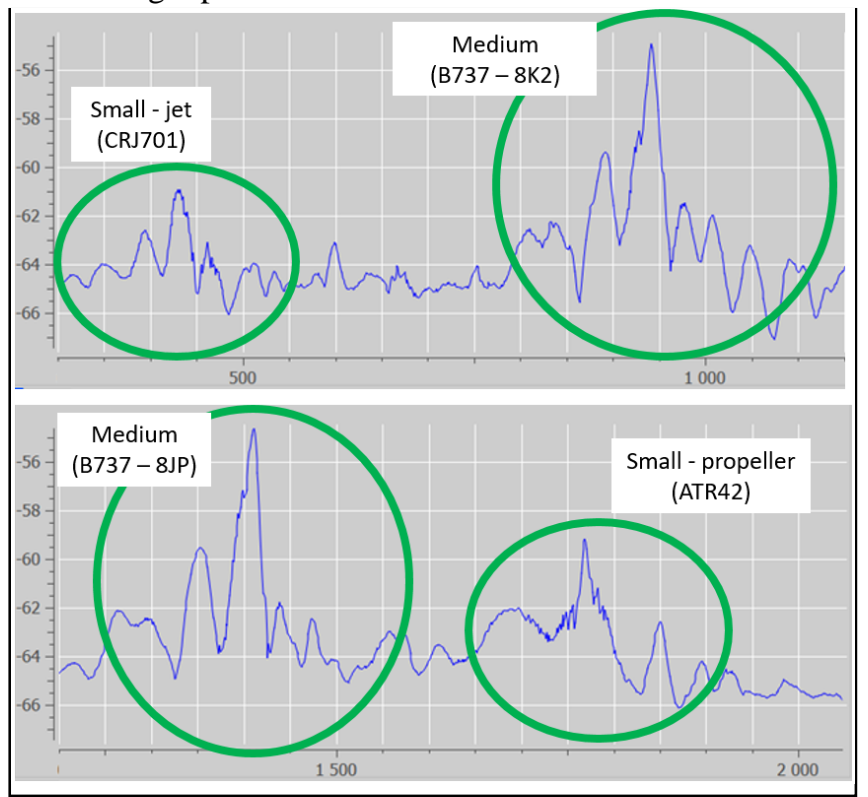

Fig. 6. Received relative level $(\mathrm{dB})$ of power, $\mathrm{HH}$, after pre-processing of reflected signal on airplanes approaching Orly-airport. Two categories are present: top with small (jet CRJ701) and medium size (Boeing 737) airplane; bottom with medium (Boeing 737) and small size airplane (airplane with propeller ATR-42 500).

It is interesting to note that, for the case of Boeing 737 from Fig. 6 which is comparable to the category medium (red line in Fig. 5, see also Tab. I) for the simulations, the difference of level in $\mathrm{dB}$ between the peak $(-62 \mathrm{~dB}$, relative) at the left side of the green circle and the maximum peak ($57 \mathrm{~dB}$, relative) is about $7 \mathrm{~dB}$, which is similar to the difference of levels for the category medium (red line) in Fig. 5 (see peak at $3.2 \mathrm{~min},-80 \mathrm{dBsm}$ and peak at $3.8 \mathrm{~min},-72$ dBsm).

TABLE II. CNN ARCHITECTURE

\begin{tabular}{|c|c|}
\hline Input Size & $(181 \times 361)$ \\
\hline \multirow{3}{*}{$\begin{array}{c}\text { Convolutional } \\
\text { layers }\end{array}$} & $\begin{array}{c}\text { Conv (6x6 (x32 channels)), ReLu MaxPool (3x3) } \\
\text { and dropout }(0.5)\end{array}$ \\
\hline & $\begin{array}{c}\text { Conv (6x6 (x64 channels)), ReLu MaxPool (3x3) } \\
\text { and dropout }(0.5)\end{array}$ \\
\hline & $\begin{array}{c}\text { Conv (6x6 (x128 channels)), ReLu MaxPool (3x3) } \\
\text { and dropout }(0.5)\end{array}$ \\
\hline \multirow{2}{*}{$\begin{array}{c}\text { Fully } \\
\text { connected } \\
\text { layers }\end{array}$} & Fully connected (256), ReLu \\
\hline & Fully connected (3), Softmax \\
\hline Output & 3 \\
\hline
\end{tabular}




\section{RESULTS OF CLASSIFICATION WITH CNN}

Similar to section II.C, we collected 269 trajectories (using ADS-B data) over which the bistatic RCS are synthesized for each trajectory, 3 categories (or classes) of plane sizes, and 11 VORs (around Paris). A natural representation of these data is considering images of RCS values vs. bistatic and aspect angles [4][5]. Since CNN has usually shown good results for image classification (AlexNet [6], VGG [7], GoogleNet [8], ResNet [9]), we propose a similar architecture (see Tab. II for details). In order to train the CNN, a 2D image (bistatic and aspect angle, $181 \times 361$ pixels, 1 pixel per angle) was constructed using the RCS variations for each trajectory and for the 11 VORs (see Fig. 7) where the colors represent the bistatic RCS values in $\mathrm{dBsm}$ (for VV polarization) for each coordinate. Preliminary results considering a single VOR at a time gave very low successful rates of classification due to the high sparsity of the images.

The blue zones in Fig. 7 correspond to the absence of ADS-B data (hence, no RCS is attributed to those coordinates). In this way a total of 807 images (269 trajectories times 3 categories) was fed to the CNN. The dataset was randomly splitted in 3 parts: training dataset (70\%), validation dataset (15\%) and test dataset (15\%). Tab. III shows the confusion matrix for the classification of validation and data set (242 samples). From this table, recognition of small class of airplanes seems better than medium and large class.

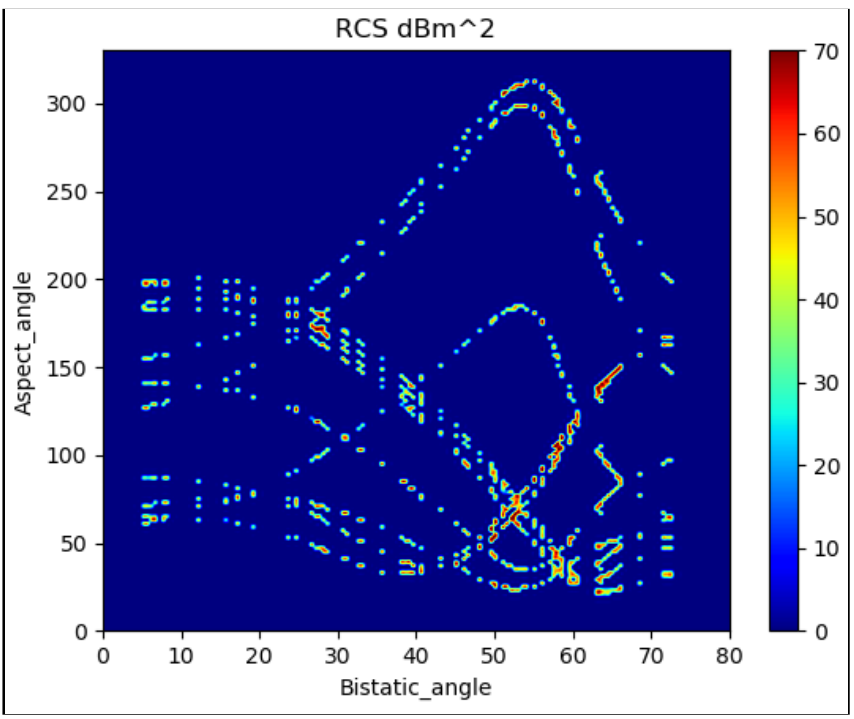

Fig. 7. 2D image of bistatic RCS variations (in color) for bistatic and aspect angles, $181 \times 361$ pixels (similar to [5]), for a single trajectory and 11 VORs (hence, 11 different illumination angles of the airplane).

TABLE III. CONFUSION MATRIX

\begin{tabular}{|l|c|c|c|}
\hline \multirow{2}{*}{$\begin{array}{c}\text { Predicted } \\
\text { class }\end{array}$} & \multicolumn{3}{|c|}{ Actual Class } \\
\cline { 2 - 4 } Small & $96.2 \%$ & Medium & Large \\
\hline Medium & $3.8 \%$ & $1.2 \%$ & $0 \%$ \\
\hline Large & $0 \%$ & $14.3 \%$ & $8.7 \%$ \\
\hline
\end{tabular}

\section{CONCLUSIONS AND PERSPECTIVES}

It has been shown, through simulations, that, globally, the bistatic RCS of airplanes of different sizes (large, medium and small) are similar, which makes difficult their discrimination on the sole basis of RCS value. It has been also shown that it is more sensible to look for the angular frequency (extraction of this information can be done as in [3]) of the bistatic RCS when the airplane is moving in order to discriminate the planes by sizes (larger planes will scatter power with finer and more peaks). These simulation results, can be used to synthesize bistatic RCS evolution for arbitrary trajectories (for example based on real trajectories) and include many VORs and different positions of receiver, in order to consider multiple scenarios based on real data to train neural networks (which need a large collection of data that many times are not available by measurement) on the recognition of airplanes. Measurements have been carried out in a passive radar configuration (Tx RBT VOR, Rx near CentraleSupélec - Gif-sur-Yvette) to collect received (scattered) power from the airplanes that are landing at Orlyairport. It has been shown that, effectively, there are enough differences on the received power from the airplanes to differentiate them. It might possible to differentiate among planes of the same category. Finally, some results were obtained using a CNN architecture to classify the airplanes by sizes using the simulation results (considering 269 trajectories, 3 classes and 11 VORs). The results provide a good, and encouraging, rate of classification.

\section{REFERENCES}

[1] S. Azarian and M. Lesturgie, "Revisiting VOR transmitters: MIMO passive radar using legacy infrastructure", International Radar Conference, 13-14 Oct., Lille, France, 2014.

[2] M. Kayton and W. R. Fried, Avionic Navigation Systems, 2nd Ed., John Wiley \& Sons Inc., USA, 1997, pp. 122-127.

[3] S. Saillant, P. Dorey and S. Azarian, "Using VHF navigation aid to estimate radar cross section of airliners by inverting the radar equation", International Radar Conference, 27-31 Aug., Brisbane, DLQ, Australia, 2018.

[4] J. Pisane, S. Azarian, M. Lesturgie and J. Verly, "Automatic real-time collection of RCS of airplanes in a real bistatic low-frequency configuration using a software defined passive radar based on illuminators of opportunity". In Proceedings of the IEEE Radar Conference (2012).

[5] J. Pisane, S. Azarian, M. Lesturgie and J. Verly, "Automatic Target Recognition for Passive Radar", IEEE Trans. on Aerospace and Electronic Systems, Vol. 50, Issue 1, Jan. 2014, pp. 371-392.

[6] A. Krizhevsky, I. Sutskever and G. E. Hinton, ImageNet classification with deep convolutional neural networks. In NIPS, pp. 1106-1114, 2012.

[7] K. Simonyan and A. Zisserman. Very deep convolutional networks for large-scale image recognition. CoRR, abs/1409.1556, 2014

[8] C. Szegedy, W. Liu, Y. Jia, P. Sermanet, S. Reed, D. Anguelov, D. Erhan, V. Vanhoucke, and A. Rabinovich, "Going deeper with convolutions," arXiv:1409.4842, 2014

[9] K. He, X. Zhang, S. Ren and J. Sun, "Deep residual learning for image recognition", arXiv: 1512 03385, 2015. 